\title{
Odours reduce the magnitude of object substitution masking for matching visual targets in females
}

\author{
Amanda K. Robinson ${ }^{1,2}$ • Julia Laning ${ }^{1}$ • Judith Reinhard ${ }^{1} \cdot$ Jason B. Mattingley $^{1,3}$
}

Published online: 15 June 2016

(C) The Psychonomic Society, Inc. 2016

\begin{abstract}
Recent evidence suggests that olfactory stimuli can influence early stages of visual processing, but there has been little focus on whether such olfactory-visual interactions convey an advantage in visual object identification. Moreover, despite evidence that some aspects of olfactory perception are superior in females than males, no study to date has examined whether olfactory influences on vision are gender-dependent. We asked whether inhalation of familiar odorants can modulate participants' ability to identify briefly flashed images of matching visual objects under conditions of object substitution masking (OSM). Across two experiments, we had male and female participants $(N=36$ in each group) identify masked visual images of odour-related objects (e.g., orange, rose, mint) amongst nonodour-related distracters (e.g., box, watch). In each trial, participants inhaled a single odour that either matched or mismatched the masked, odour-related target. Target detection performance was analysed using a signal detection $(d$ ') approach. In females, but not males, matching odours significantly reduced OSM relative to mismatching odours, suggesting that familiar odours can enhance the salience of briefly presented visual objects. We conclude that olfactory cues exert a subtle influence on visual
\end{abstract}

Electronic supplementary material The online version of this article (doi:10.3758/s13414-016-1157-9) contains supplementary material, which is available to authorized users.

Amanda K. Robinson

amanda.robinson@uqconnect.edu.au

1 Queensland Brain Institute, The University of Queensland, St Lucia 4072, Australia

2 Carnegie Mellon University, Pittsburgh, PA, USA

3 School of Psychology, The University of Queensland, St Lucia 4072, Australia processes by transiently enhancing the salience of matching object representations. The results add to a growing body of literature that points towards consistent gender differences in olfactory perception.

Keywords Multisensory processing $\cdot$ Olfaction $\cdot$ Visual perception

\section{Introduction}

Human olfaction is markedly different from the sense of vision but has similarly profound effects on behaviour and cognition. Although individuals notoriously underestimate their sense of smell, the olfactory system has extremely high resolution; humans can likely distinguish more than one trillion different odorants (Bushdid, Magnasco, Vosshall, \& Keller, 2014). Odours can influence sustained attention (Warm, Dember, \& Parasuraman, 1991), concentration (Sakamoto, Minoura, Usui, Ishizuka, \& Kanba, 2005), dual task performance (Ho \& Spence, 2005), and working memory (Habel et al., 2007). In addition to these general effects on cognition, it is becoming increasingly clear that olfactory processes have unique contributions during multisensory perception. Olfactory perception is extremely important in flavour perception in particular (Zampini \& Spence, 2012) but also interacts with the nonchemical senses, including vision.

Olfaction and vision are inextricably linked; multisensory interactions between these two senses are apparent in many circumstances. Specific odours have cross-modal associations with colours (Dematte, Sanabria, \& Spence, 2006; Gilbert, Martin, \& Kemp, 1996) and shapes (Hanson-Vaux, Crisinel, \& Spence, 2013; Seo, Arshamian, et al., 2010), and these associations are consistent across individuals (Gilbert et al., 1996; Hanson-Vaux et al., 2013; Kemp \& Gilbert, 1997; 
Osterbauer et al., 2005). Such associations demonstrate olfactory-visual convergence and can be useful for studying object-based multisensory interactions. Previous studies have shown that matching visual features, such as colour and object shape, can enhance odour detection (Gottfried \& Dolan, 2003), odour discrimination (Dematte, Sanabria, \& Spence, 2009), and intensity (Zellner \& Kautz, 1990). These interactions also are bidirectional. As noted, certain visual features can enhance odour perception, but perhaps more unexpectedly, specific odours also can influence visual perception (Chen, Zhou, Chen, He, \& Zhou, 2013; Robinson, Mattingley, \& Reinhard, 2013; Zhou, Jiang, He, \& Chen, 2010). Under conditions of binocular rivalry, for example, matching odorants can prolong durations of perceptual dominance of matching versus mismatching visual objects (Zhou et al., 2010; Zhou, Zhang, Chen, Wang, \& Chen, 2012). We have shown that priming with a familiar odour can reduce the magnitude of the attentional blink for matching visual objects presented in rapid streams of distractor objects at fixation (Robinson et al., 2013). Likewise, in the spatial domain, Chen and colleagues (2013) showed that odours can reduce reaction times for images of matching objects in dot-prime and visual search paradigms. Such studies demonstrate that olfaction can influence vision even under conditions in which the experimental odours are task-irrelevant.

In addition to this behavioural evidence for olfactory-visual interactions, neuroimaging studies have shed light on the underlying neural processes involved. In a recent study, we used EEG to investigate how odours influence perception of matching and nonmatching visual objects as participants completed an orthogonal visual task. In females, odours enhanced the amplitude of the $\mathrm{N} 1$ component in response to matching images approximately $140 \mathrm{~ms}$ after image presentation. Congruency effects were apparent regardless of whether the objects were coloured or greyscale, indicating that odours might influence object identification mechanisms (Robinson, Reinhard, \& Mattingley, 2015). Gottfried and Dolan (2003) found that matching odour-image pairs resulted in greater activation in the orbitofrontal cortex (OFC) relative to nonmatching combinations, and this OFC activity correlated with faster odour detection. Their study was primarily concerned with activity in olfactory areas of the brain; functional connections between olfactory and visual regions of the brain remain unknown. Given the evidence that olfaction can influence early visual processing, it seems conceivable olfactoryvisual interactions might involve occipital cortex. Furthermore, olfactory-visual interactions might plausibly be influenced by the features and functions of both the olfactory and visual sensory systems.

Olfactory perception, unlike the other senses, differs markedly between females and males. Females outperform males on tasks of odour discrimination, identification, and memory (Choudhury, Moberg, \& Doty, 2003; Larsson, Nilsson,
Olofsson, \& Nordin, 2004; Zucco, Aiello, Turuani, \& Koster, 2012), pointing to gender differences in higher order olfactory tasks involving verbal abilities. Low-level olfactory perception also might differ between males and females. Females reportedly have lower olfactory thresholds than males (Cometto-Muniz \& Abraham, 2008; Koelega, 1970), although several studies have reported no difference in odour sensitivity between males and females (Lundstrom \& Hummel, 2006; Oberg, Larsson, \& Backman, 2002). Gender differences have been reported for the trigeminal system (Andersson, Lundberg, Astrom, \& Nordin, 2011), which is stimulated by most odours. In contrast, few gender differences have been documented for perception in other modalities (Fairweather, 1976; Sabatinelli, Flaisch, Bradley, Fitzsimmons, \& Lang, 2004). Surprisingly, there has been little focus on gender differences in multisensory integration between olfaction and other modalities, despite accumulating research on interactions between olfaction and vision. Several olfactory-visual experiments have been conducted with a majority of female participants (Chen et al., 2013; Seo, Roidl, Muller, \& Negoias, 2010; Seo, Arshamian, et al., 2010; Zhou et al., 2012), so it is possible that previous results have been driven by larger multisensory effects in females than males.

Recently, we demonstrated that odours enhanced the N1 component of visual event-related potentials (ERPs) in females but not males (Robinson et al., 2015). Such results are consistent with differences in the anatomical structure of the olfactory system in females and males. For example, GarciaFalgueras and colleagues (2006) found marked gender differences for grey matter density in the olfactory system, suggesting that olfaction is a sexually dimorphic modality. Specifically, females had greater grey matter density in areas of OFC, amygdala, and hippocampus, whereas males had greater density in entorhinal cortex and another region of the OFC (Garcia-Falgueras et al., 2006). Likewise, Frasnelli and colleagues (2010) found that females' odour discrimination and identification ability is positively correlated with grey matter thickness in the intraparietal sulcus (IPS) and lingual gyrus, both areas involved in high-level visual processing (Grefkes \& Fink, 2005; Haxby, Hoffman, \& Gobbini, 2000; Sergent, Ohta, \& Macdonald, 1992), whereas these abilities are negatively correlated with cortical thickness in the same regions in males (see also Seubert, Freiherr, Frasnelli, Hummel, \& Lundstrom, 2013). Sexual dimorphism within the olfactory system is likely to differentially influence olfactory-visual multisensory perception, but to our knowledge no study has investigated how interactions between olfaction and vision manifest differently in males and females.

In the current study, we examined the influence of familiar odours on visual identification under conditions of object substitution masking (OSM; Enns \& Di Lollo, 1997). In a typical OSM paradigm, participants are asked to discriminate a target that is surrounded by a four-dot mask (Enns \& Di Lollo, 
1997). If the mask remains after the object is briefly presented, participants typically report seeing the mask but find it difficult to discriminate the target object. If the mask disappears at the same time as the object, there is no such deficit in object discrimination. According to one prominent theory, OSM occurs due to a conflict between the target object and the mask at different stages of visual processing, reflecting re-entrant processing between primary visual cortex (V1), other areas of occipital cortex and higher cortical areas in the temporal and parietal lobes (Di Lollo, Enns, \& Rensink, 2000; Dux, Visser, Goodhew, \& Lipp, 2010). Another account, the object updating hypothesis, suggests that OSM is caused by a failure in individuation of the target and the mask (Lleras \& Moore, 2003; Pilling \& Gellatly, 2010), possibly due to limitations in the temporal resolution of visual encoding (Goodhew, Pratt, \& Dux, 2013). Carlson, Rauschenberger, and Verstraten (2007) found that neural responses to repeated targets subject to OSM reflected adaptation in V1 but not lateral occipital cortex, suggesting the neural representation of the masked target object was reduced, if not completely suppressed, in ventral occipital cortex. Crucially, an ERP study found early stages of visual processing were intact during OSM, while processing stages after $130 \mathrm{~ms}$ were reduced, and object-specific processing from $180 \mathrm{~ms}$ was eliminated (Harris, $\mathrm{Ku}$, \& Woldorff, 2013), supporting the idea that OSM reduces object representations. There is evidence, however, that masked target objects can support semantic perception (Goodhew, Visser, Lipp, \& Dux, 2011) and action (Heath, Maraj, Godbolt, \& Binsted, 2008), despite not reaching awareness. These lines of evidence suggest ventral object representations might be necessary for awareness.

Previous studies have shown that congruent odours influence discrimination of visual objects during binocular rivalry and the attentional blink (Robinson, Mattingley, \& Reinhard, 2013; Zhou et al., 2010; Zhou, Jiang, He, \& Chen, 2010), but these paradigms have been shown to reflect bottlenecks at different levels of processing than OSM. Specifically, OSM seems to influence object representations despite initial intact processing of objects. We reasoned that the OSM paradigm would be a good candidate for investigating whether odours affect discrimination of familiar visual objects associated with a characteristic odour. In this study, we asked whether familiar odorants, such as orange and mint, can alter participants' ability to detect matching visual objects, such as images orange or mint leaves, under conditions of OSM. Given previous findings that such odours can enhance the perceptual salience of matching visual objects (Chen et al., 2013; Robinson et al., 2013), we predicted that OSM would be reduced for matching odour-object pairs relative to mismatching pairs. We also examined whether olfactory-visual OSM performance differed for males and females by recruiting and testing a large cohort of female $(\mathrm{N}=36$; Experiment 1$)$ and male $(\mathrm{N}=36$; Experiment 2) participants. Based on previous evidence, we predicted greater olfactory modulation of visual OSM in females.

\section{Materials and methods}

\section{Participants}

This study was approved by the Human Research Ethics Committee of The University of Queensland, in accordance with the Australian National Health and Medical Research Council's guidelines. Informed consent was obtained from all participants. Experiments 1 and 2 consisted of 36 female participants ( $M=24.42$ years, $S . D .=5.42$ years $)$ and 36 male participants $(M=26.81$ years, $S . D .=6.24$ years $)$, respectively. The experiments were otherwise identical. The sample size was chosen based on larger sample sizes from previous olfactory-visual experiments to allow sufficient power to detect real effects. Power analysis using $G^{*}$ power indicated a sample size of 34 participants would be needed to detect medium pairwise effects $(\mathrm{d}=0.5)$ with $80 \%$ power at alpha $=$ 0.05 .

All participants were recruited from The University of Queensland. They were screened for their ability to distinguish between a set of test odours, as described below, and completed a questionnaire relating to factors, such as age, odour allergies, and whether they were taking hormonerelated medications. All participants reported normal olfactory perception, normal or corrected-to-normal vision, were nonsmokers, and had no known odour allergies.

\section{Design and stimuli}

The participants' task was to detect the presence of a masked, odour-related target image (rose, orange, or mint) amongst a set of non-odour-related distracter images (e.g., box, hat). Immediately prior to the onset of the visual display, participants inhaled a single odorant (rose, orange, mint, or plain air).

We employed an OSM paradigm with two target conditions (target present, target absent), two masking conditions (simultaneous offset mask, delayed offset mask), and three odour conditions (odour congruent with target, odour incongruent with target, no odour). On every trial, four visual objects were shown concurrently, one in each quadrant of the display and positioned $8^{\circ}$ from a central fixation cross. For $50 \%$ of trials, there was one target image and three distracter images (target-present trials), and for the other $50 \%$ of trials, there were four distracter images (target-absent trials). A black, four-dot mask surrounded the target. Each dot was a $0.35^{\circ}$ diameter circular disk and the four dots were arranged as the corners of a $3.18^{\circ} \times 3.18^{\circ}$ square. On nontarget trials, the dot-mask surrounded one of the distracters. Only one dotmask was displayed on any given trial. 
The visual target was always an odour-related object (images of an orange, a rose or mint leaves; Figs. 1 and S1). There were seven different exemplars of each target object. The nontarget distracter images were randomly selected from a set of 16 objects that do not have a typical odour (e.g., hat, tree, basketball; Figure S2). All images were achromatic (greyscale) and each was sized to fit within a $3 \times 3$ imaginary square. The images were displayed on a uniform grey background (RGB values: 127, 127, 127). The Cogent Toolbox in Matlab was used to present the visual stimuli on a 21 -inch, Dell P1130 CRT monitor with a display resolution of $1024 \times$ 768 pixels and $100-\mathrm{Hz}$ refresh rate. Participants placed their chin on a chin rest $60 \mathrm{~cm}$ from the monitor.

The odours used were orange, mint (Raindrops Essential Oils), and rose (Queens Fine Foods flavouring essence). As shown in Fig. 1, there were three visual-olfactory conditions. In each block, participants were instructed to look for a particular visual target (orange, rose, or mint). On each trial, they were asked to inhale an odour and detect whether the target image (e.g., orange) was present or absent at the location of the mask. On target-absent trials, a distracter object appeared at the masked location. The odour was either congruent with the visual target, incongruent with the visual target, or was plain air (visual-only condition).

Each participant was assigned two of the three possible target objects (i.e., rose and orange; rose and mint; or orange and mint) for the duration of the experiment. Across the group, equal numbers of participants completed each of the three possible odour-image pairs. Trials were blocked by masking condition and target type. There were eight blocks in the experiment, two blocks for each masking condition ("masked" - delayed offset mask, and "unmasked" - simultaneous offset mask) and target types (two of orange, rose, and mint). In each block, there were 72 trials. Trials were randomised within each block and blocks were randomised throughout the experiment. In total, there were 96 trials for each combination of odour condition (congruent, incongruent and no odour) and target condition (target and nontarget). Participants were offered rest breaks in between blocks. An experimental session took approximately 50 minutes.

Participants were instructed to look for a visual target (orange, rose, or mint). On each trial, they had to inhale an odour and detect whether the target object (e.g., orange) was present or absent at the location of the mask. On target-absent trials, a distracter object appeared at the masked location. The odour was either congruent with the visual target, incongruent with the visual target, or was plain air (visual-only condition). All visual stimuli were shown in greyscale.

\section{Procedure}

Odour discrimination test Participants first completed a three-alternative, forced-choice odour discrimination task. Three different odours were presented to participants: two critical odours (two of rose, mint, and orange, as used in the main experimental task) and plain air (no odour). Each odour was presented 5 times, and the 15 trials were randomised. On each trial, participants were given 3 seconds to prepare for inhalation, after which the odour was presented for 4 seconds. Participants pressed a button to indicate which odour they perceived. The next trial began when participants made their response.

Target detection task Figure 2 shows an example trial for the masked and unmasked conditions of the olfactory-visual OSM task. At the start of each trial, a fixation cross appeared centrally, and simultaneously a tone was presented as a cue for the participants to inhale through their nose. A four-channel olfactometer delivered odours to participants by driving clean air at 1.5 litres per minute through bottles containing undiluted

\begin{tabular}{|c|c|c|c|c|c|}
\hline \multirow{2}{*}{ Target } & \multicolumn{2}{|c|}{ Masked object } & \multicolumn{3}{|c|}{ Odour condition } \\
\hline & Target & Non-target & $\begin{array}{l}\text { Congruent } \\
\text { odour-target }\end{array}$ & $\begin{array}{l}\text { Incongruent } \\
\text { odour-target }\end{array}$ & Visual-only \\
\hline Orange & & & Orange & Rose, Mint & No odour \\
\hline Rose & & & Rose & Orange, Mint & No odour \\
\hline Mint & & & Mint & Orange, Rose & No odour \\
\hline
\end{tabular}

Fig. 1 Experimental design 
Masked condition

(delayed dot offset)

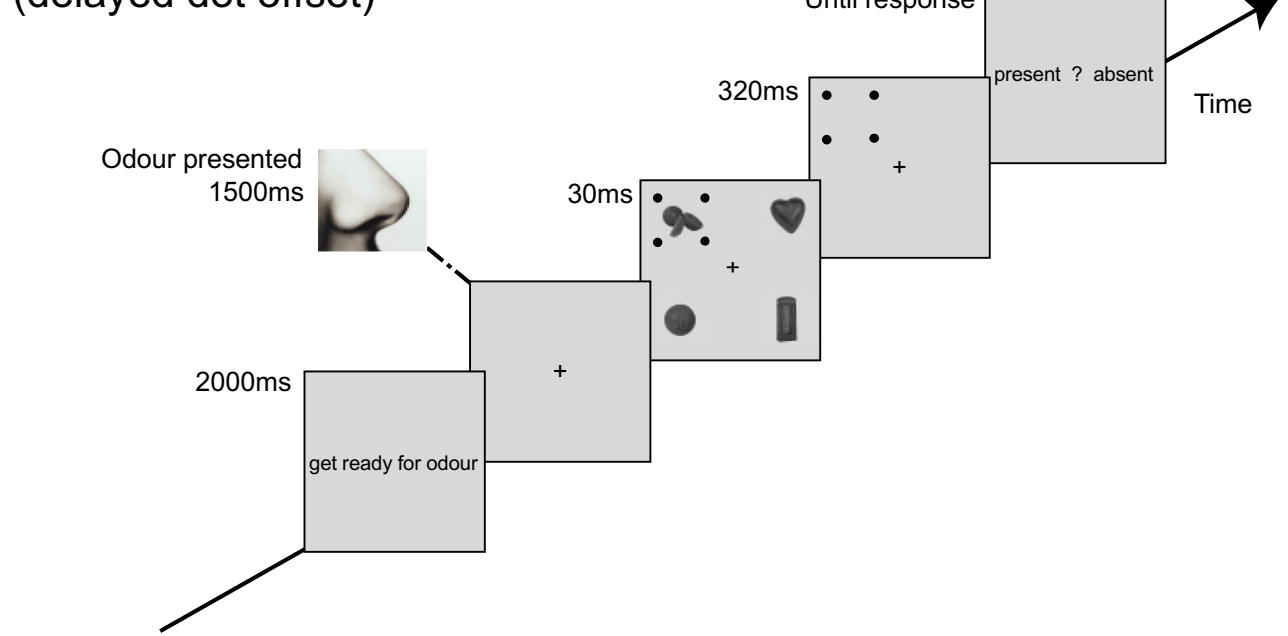

Unmasked condition

(simultaneous dot offset)

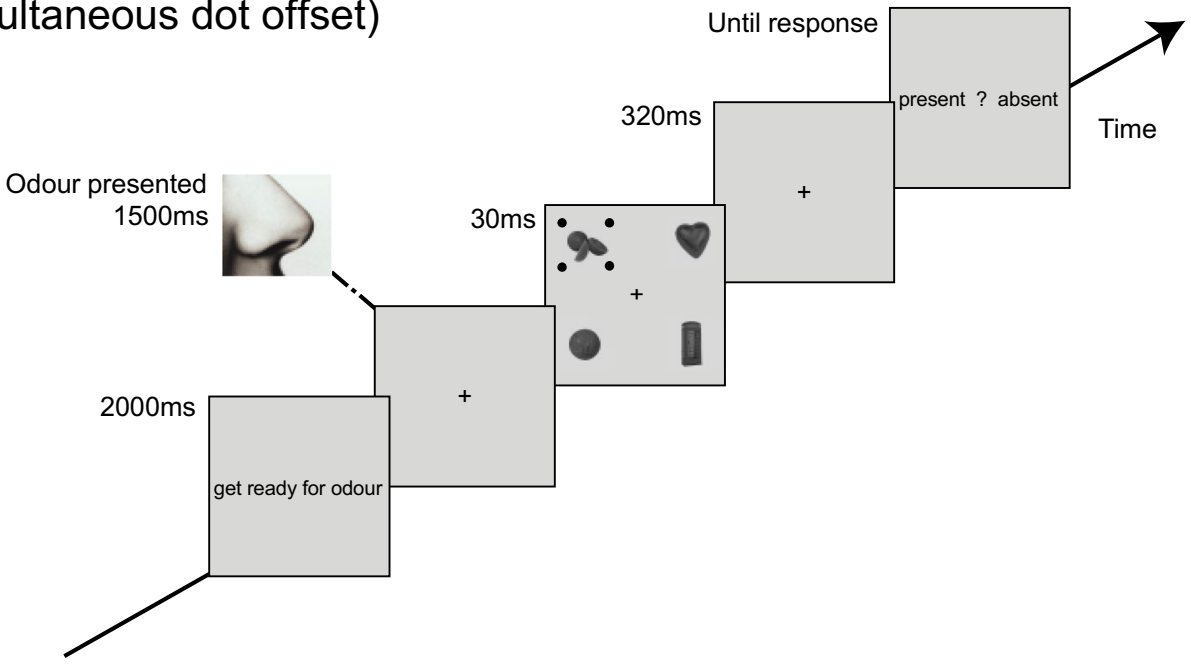

Fig. 2 Example trials for the masked and unmasked conditions of the olfactory-visual OSM experiment

odour solution. Odours from the four lines emerged at a funnel placed in front of a chin rest, under the participants' nose. The odour was presented for $1500 \mathrm{~ms}$. The four visual objects and single four-dot mask were then displayed for $30 \mathrm{~ms}$. The mask cued participants to report on the target object within the corresponding quadrant of the display. In the simultaneous offset condition, the dots disappeared at the same time as the four images. In the delayed offset condition, the dots remained visible for another $320 \mathrm{~ms}$ after the four images disappeared. Participants were asked to indicate whether the object at the location of the mask was the designated target ("present") or a nontarget ("absent"), by pressing the left or right arrow keys with the index or middle finger of their right hand, respectively.

Distracter (nontarget) objects appeared in the remaining three (nonmasked) locations. Participants had to inhale an odour and then detect whether the masked image was the target or a nontarget. Within each block, odours could be either congruent or incongruent with the target object, with equal probability. Plain air (no odour) was used as a baseline.

\section{Data analysis}

Target detection sensitivity $(d$ ') was calculated for each of the masking and congruency conditions per participant and was averaged across participants. A small number of individuals were excluded from the analyses because their $d$ ' scores were below 0.1 for the delayed offset condition $(N=2$ females in Experiment 1;N=1 male in Experiment 2). Inspection of the data revealed that these participants performed well below chance for target-present trials $(\leq 37.50 \%$ hits, $N=1$ female, 1 male) or target absent trials (32.64\% correct rejection, $N=1$ female), reflecting a strong bias for absent and present responses, respectively. The pattern of results described below 
did not change if data from these participants were included in the analysis. Two participants correctly detected the target on all trials in one condition (hit rate $=1$ ), meaning $d$ ' could not be computed, so their hit rates were calculated by $(n-0.5) / n$, where $n$ is the number of target-present trials (Stanislaw \& Todorov, 1999).

For each experiment, a $3 \times 2$ within-subjects analysis of variance (ANOVA) was conducted to determine the effects of congruency (congruent, incongruent, no odour) and masking (simultaneous versus delayed offset) on visual sensitivity, as indexed by $d$ '. Significant interactions between congruency and masking were followed up by investigating the influence of odours on masking magnitude, which was calculated as the difference in sensitivity between the simultaneous- and delayed mask-offset conditions. An $\alpha$ level of 0.05 was used for all statistical tests.

As a complement to null hypothesis significance testing, the effects of odour congruency on visual masking were assessed using Bayesian analyses to quantify the strength of evidence for both null and alternative hypotheses (Morey \& Rouder, 2011). Bayes factors were calculated for all pairwise comparisons using the BayesFactor package in R. A Bayes factor $(B)$ represents the strength of support for the alternative hypothesis (H1) relative to the null; values of $B>3$ suggest substantial evidence in favour of $\mathrm{H} 1$, whereas values smaller than one third are regarded as providing substantial evidence in favour of the null hypothesis (Dienes, 2011; Jeffreys, 1961). Bayesian statistics were performed on the critical hypotheses, namely, that a congruent odour should reduce masking magnitude relative to the incongruent odour ( $B_{\text {(congruent }}<$ incongruent) $)$ and no odour $\left(B_{\text {(congruent }<\text { no odour })}\right)$ conditions and that an incongruent odour should enhance masking relative to the no odour condition $\left(B_{\text {(no odour }<\text { incongruent) })}\right) . B$ statistics also were reported for effects in the opposite direction.

Effect sizes were calculated for all statistical comparisons. Generalised eta-squared values $\left(\eta_{\mathrm{g}}{ }^{2}\right)$ were calculated for ANOVA and ANCOVA results, and Hedges' $g_{\text {av }}$ or $g_{\mathrm{s}}$ were calculated for paired and independent samples $t$ tests, respectively (Bakeman, 2005; Lakens, 2013).

\section{Results}

\section{Odour discrimination test}

In the odour discrimination task performed prior to the experimental task, female participants correctly identified the odour on $91.0 \%$ of trials (S.E.M. $=1.6 \%$ ), significantly higher than chance $(33.3 \%), t(33)=35.92, p<0.001$. Males correctly identified the odour on $85.1 \%$ of trials (S.E.M. $=1.8 \%$ ), also significantly above chance, $t(34)=29.41, p<0.001$. Performance was significantly higher in females than males, $t(67)=2.43, p=0.018, g_{\mathrm{s}}=0.579, B_{(\text {females }>\text { males })}=5.77$,
$B_{\text {(males }>\text { females) }}=0.08$, indicating that females were better at discriminating the test odours than males.

\section{Experiment 1 - Female participants}

Overall, for target-present trials, females $(N=34)$ correctly detected the target image in $68.3 \%$ (S.E.M. $=2.8 \%)$ of trials in the delayed-offset condition and on $86.3 \%($ S.E.M. $=1.5 \%)$ of trials in the simultaneous-offset condition. For target-absent trials, they responded correctly on $73.3 \%($ S.E.M. $=2.24 \%)$ of trials in the delayed-offset condition and in $78.3 \%$ (S.E.M. $=$ $1.9 \%$ ) in the simultaneous-offset condition. Sensitivity measures revealed $d$ ' values of 1.23 for delayed-offset trials and 2.04 for simultaneous-offset trials.

Figure $3 \mathrm{a}$ shows the mean difference in the magnitude of the masking effect (i.e., the difference in $d$ ' between the simultaneous- and delayed-offset conditions) for the three odour conditions (congruent, incongruent, no odour). The masking effect for female participants was greatest for the incongruent condition, somewhat lower for the no-odour condition, and smallest for the congruent condition. A $3 \times 2$ ANOVA was performed to assess the impact of odour-image congruency and masking condition on $d$ ' scores. The analysis revealed a significant main effect of masking condition, $F(1$, $33)=90.30, p<0.001, \eta_{\mathrm{g}}{ }^{2}=0.359$, such that sensitivity in the delayed-offset condition $(M=1.23)$ was significantly lower than that for the simultaneous-offset condition $(M=2.04)$. There was no significant main effect of congruency, $F(2,66)$ $=0.78, p=0.462, \eta_{\mathrm{g}}{ }^{2}=0.002$, but there was a significant interaction between congruency and masking, $F(2,66)=$ $3.80, p=0.028, \eta_{\mathrm{g}}{ }^{2}=0.012$. To investigate this significant interaction, the effect of odour condition on masking magnitude was assessed using paired $t$ tests with a Bonferroni correction $(\alpha=0.017)$. Masking magnitude in the congruent

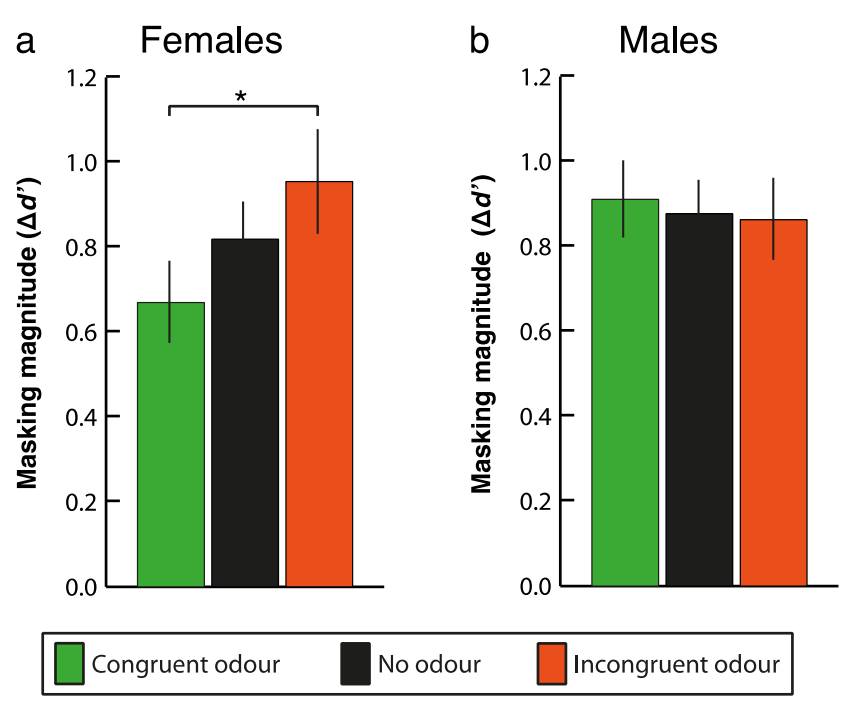

Fig. 3 Masking magnitude across odour condition for Experiments 1 and 2 
odour condition was significantly lower than in the incongruent odour condition, $t(33)=-2.76, p=0.009, g_{\mathrm{av}}=0.444$, $B_{\text {(congruent }<\text { incongruent) }}=9.12, B_{\text {(congruent }>\text { incongruent })}=0.05$. The common language effect size indicates that after controlling for individual differences, the likelihood that a congruent odour will reduce masking relative to an incongruent odour is $68.2 \%$. There was no significant difference between the noodour condition and the congruent odour condition, $t(33)=$ $1.61, p=0.118, g_{\mathrm{av}}=0.271, B_{\text {(congruent }<\text { no odour })}=1.10$, $B_{\text {(congruent }>\text { no odour) }}=0.08$, or between the no-odour and incongruent odour conditions, $t(33)=-1.21, p=0.233, g_{\text {av }}$ $=0.226, B_{\text {(no odour }<\text { incongruent })}=0.63, B_{\text {(no odour }>\text { incongruent })}=$ 0.09 .

Masking magnitude was calculated as the difference in $d^{\prime}$ between the simultaneous- and delayed-offset conditions; a larger masking effect reflects a greater decrement in the delayed-offset condition relative to the simultaneous offset condition. a) In females, a congruent odour significantly reduced masking relative to an incongruent odour. b) In males, there was no effect of odour congruency on masking magnitude. ${ }^{*} p<0.05$. Error bars represent one standard error of the mean.

\section{Experiment 2 - Male participants}

For target-present trials, males $(N=35)$ correctly detected the target object on $65.9 \%$ (S.E.M. $=2.7 \%)$ of trials in the delayed-offset condition and $84.8 \%$ (S.E.M. $=1.2 \%)$ of trials in the simultaneous-offset condition. For target-absent trials, they responded correctly on $68.7 \%$ (S.E.M. $=2.0 \%$ ) of trials in the delayed-offset condition and on $75.8 \%$ (S.E.M. $=1.60 \%$ ) of trials in the simultaneous-offset condition. Sensitivity calculations revealed $d$ ' values of 0.97 and 1.86 for the delayedand simultaneous-offset conditions, respectively.

Figure $3 \mathrm{~b}$ shows the mean difference in the magnitude of the masking effect for the congruent, incongruent and noodour conditions. Masking magnitude did not differ across the three odour conditions. A $3 \times 2$ ANOVA on sensitivity scores revealed a significant main effect of masking condition, $F(1,34)=168.90, p<0.001, \eta_{\mathrm{g}}{ }^{2}=0.377$, such that sensitivity in the delayed-offset condition was significantly lower than in the simultaneous-offset condition. However, there was no significant main effect of congruency, $F(2,68)=1.85, p=0.165$, $\eta_{\mathrm{g}}{ }^{2}=0.006$, and no significant interaction between congruency and masking, $F(2,68)=0.17, p=0.845, \eta_{\mathrm{g}}{ }^{2}<0.001$. To assess the evidence that odour congruency had no effect on masking magnitude, pairwise comparisons were performed for each combination of odour conditions, as was done in Experiment 1. There was no difference between the congruent and incongruent conditions, $t(34)=0.69, p=0.494, g_{\mathrm{av}}=$ $0.100, B_{\text {(congruent }<\text { incongruent) }}=0.12, B_{\text {(congruent }>\text { incongruent) }}=$ 0.34 , the no odour and congruent conditions, $t(34)=-0.37, p$ $=0.712, g_{\text {av }}=0.082, B_{\text {(congruent }<\text { no odour) }}=0.14, B_{\text {(congruent }>\text { no }}$ odour) $=0.25$, or the no-odour and incongruent conditions, $t(34)=-0.13, p=0.895, g_{\mathrm{av}}=0.026, B_{\text {(no odour }<\text { incongruent) }}$ $=0.16, B_{\text {(no odour }>\text { incongruent) }}=0.20$. Overall, Bayes analyses revealed substantial evidence in favour of the null hypothesis, indicating that odours had no effect on masking of visual images in males.

\section{Comparison of results from experiments 1 and 2}

To directly compare the results of Experiments 1 and 2, we conducted a $3 \times 2 \times 2$ ANOVA to investigate the effect of odour-target congruency, masking and gender on target sensitivity. Analyses revealed a significant effect of gender, $F(1,67)$ $=4.02, p=0.049, \eta_{\mathrm{g}}{ }^{2}=0.037$, such that target-detection sensitivity was lower overall for males $(M=1.42)$ than females $(M=1.64)$. As expected, there was also a significant main effect of masking, $F(1,67)=242.75, p<0.001, \eta_{\mathrm{g}}{ }^{2}=$ 0.368 , such that sensitivity was lower in the delayed-offset condition than in the simultaneous-offset condition. There was no significant main effect of congruency, $F(2,134)=$ $1.28, p=0.282, \eta_{\mathrm{g}}{ }^{2}=0.002$, and no significant two-way interactions between congruency and gender, $F(2,134)=$ $1.57, p=0.213, \eta_{\mathrm{g}}{ }^{2}=0.002$, masking and gender, $F(1,67)=$ $0.40, p=0.528, \eta_{\mathrm{g}}{ }^{2}=0.001$, or congruency and masking, $F(2$, $134)=1.26, p=0.286, \eta_{\mathrm{g}}{ }^{2}=0.002$. There was, however, a marginal three-way interaction between congruency, masking, and gender, $F(2,134)=2.86, p=0.061, \eta_{\mathrm{g}}{ }^{2}=0.004$. This result reflected the effect of odour-image congruency on masking magnitude in females but not males.

As outlined earlier, females performed better than males in the olfactory discrimination task. To determine whether olfactory discrimination ability, rather than gender per se, might have been responsible for the difference in the magnitude of masking between the two groups, we conducted an analysis of covariance (ANCOVA) on masking magnitude with factors of odour condition (congruent, incongruent, no odour) and gender (male, female), and the covariate of odour discrimination score. There were no significant main effects of odour condition, $F(2,130)=1.24, p=0.293, \eta_{\mathrm{g}}^{2}=0.007$, gender, $F(1,65)$ $=0.40, p=0.532, \eta_{\mathrm{g}}{ }^{2}=0.004$, or odour discrimination score, $F(1,65)=0.27, p=0.607, \eta_{\mathrm{g}}{ }^{2}=0.003$. Furthermore, there were no significant interactions between odour condition and odour discrimination score, $F(2,130)=0.55, p=0.580$, $\eta_{\mathrm{g}}{ }^{2}=0.003$, gender and odour discrimination score, $F(1,65)=$ $0.43, p=0.516, \eta_{\mathrm{g}}{ }^{2}=0.004$, or odour condition, gender, and odour discrimination score, $F(2,130)=0.23, p=0.793, \eta_{\mathrm{g}}{ }^{2}=$ 0.001. A marginal interaction between odour condition and gender remained, $F(2,130)=2.81, p=0.064, \eta_{\mathrm{g}}{ }^{2}=0.016$, indicating that odour discrimination ability did not account for the observed odour congruency effect in females.

To summarise, we found that in females, but not males, familiar odours reduced OSM for matching visual images 
relative to mismatching images. This effect was not associated with olfactory discrimination ability.

\section{Discussion}

This study was designed to test the hypothesis that an odour can enhance detection of a matching visual object during object substitution masking (OSM) and that this effect would be larger in females than males. We found that a congruent odour reduced the magnitude of masking relative to an incongruent odour, but only in females. These findings show that sense of smell can enhance visual discrimination for matching olfactory-visual object pairs. Essentially, it seems odours prime visual representations of matching objects, thus making them more salient and more difficult to mask. Previous studies have shown that olfactory-visual integration serves to enhance perception when olfactory and visual information is congruent (Chen et al., 2013; Robinson et al., 2013). The current study builds on these previous findings by showing that cross-modal associations between odours and their corresponding visual objects can enhance object identification when visual awareness is disrupted. Furthermore, the results suggest that the olfactory and visual systems might be functionally more tightly linked in females than males.

This study was designed to investigate whether odourimage congruency influences perception differentially for males and females. Overall, congruent odours significantly reduced masking relative to incongruent odours in females, whereas this effect was absent in males. In the additional analysis comparing the results of Experiments 1 and 2, the threeway interaction between odour congruency, masking, and gender was not significant $(p=0.061)$, but the direction of the trend was as expected and was confirmed by the planned analyses within the two individual experiments. Moreover, Bayesian statistics, typically more conservative than traditional null hypothesis tests, revealed that there was enough evidence to conclude that odours exerted no effect on visual masking in males. This is a striking result, because no previous studies have reported a gender difference for OSM, indicating this effect is not due to a difference in visual perception alone. Females in the study were in fact more sensitive to the visual targets overall, as reflected in their larger mean d-prime values, than males, ruling out any potential ceiling effects in males. It seems logical that the gender differences arose from differences in the olfactory system. Crucially, however, olfactory ability as measured in this study did not account for differential effects of odour condition on masking for females and males. Therefore, multisensory integration between olfaction and vision evidently manifests differently in males and females, perhaps reflecting different neural mechanisms for olfactory perception. Many studies have noted differences in olfactory preferences between males and females; for example, women prefer the scent of MHC-dissimilar men (Wedekind \& Furi, 1997; Wedekind, Seebeck, Bettens, \& Paepke, 1995). It could be that women are more motivated to draw links between odours and visual objects for purposes, such as sexual selection, which leads to stronger connections between olfactory and visual areas of the brain than males. As noted earlier, a previous study found that grey matter thicknesses in IPS and lingual gyrus were positively correlated with olfactory abilities in females but not males (Frasnelli et al., 2010). Furthermore, in females odours enhanced early visual processing as indexed by ERPs (Robinson et al., 2015). The specific mechanism for this gender difference remains to be determined, but we suggest that females might have stronger functional connections between the olfactory and visual systems in the brain, leading to better associations between odours and visual features of objects.

Our observation of reduced masking in females with congruent relative to incongruent odours can potentially elucidate the stages of perceptual processing over which visual and olfactory inputs interact in the brain. During object substitution masking, early feed-forward processing remains intact, and the earliest modulation of neural activity is observed at around 130-170 ms, with a slight reduction due to masking (Harris et al., 2013). Importantly, however, object categoryspecific activity, as indexed by a face-house comparison observed in the N170 ERP component, is completely eliminated by OSM (Carlson et al., 2007; Harris et al., 2013; Reiss \& Hoffman, 2007). A recent study found OSM does not require distributed spatial or temporal attention, suggesting mechanisms of OSM are not related to attentional processes (Filmer, Mattingley, \& Dux, 2015). In the current study, it is likely that congruent odours influenced a relatively early perceptual stage of visual processing not disrupted during OSM. Previous behavioural studies have suggested that olfaction and vision are integrated at an early stage of perceptual processing (Seo, Arshamian, et al., 2010; Zhou et al., 2010, 2012), and ERPs clearly show that odours enhance processing of matching images at the N1 component in females, peaking at approximately $135 \mathrm{~ms}$ after image presentation (Robinson et al., 2015).

The current results are consistent with the notion that odours influence re-entrant visual processing. It could be that odours enhance the salience of matching images such that early feed-forward processes are stronger and the objects more resilient to masking. Alternatively, odours might directly enhance recurrent processes. More specifically, visual re-entrant processes supporting conscious perception might be influenced by matching inputs from other sensory modalities, in this case olfaction. Interestingly, awareness in OSM has been linked to re-entrant activation of primary visual cortex (V1) from higher visual areas (Boehler, Schoenfeld, Heinze, \& Hopf, 2008). Odours might therefore enhance the representation of matching visual objects within occipital brain regions, 
thereby allowing masked images to reach awareness (at least in females).

The results from Experiment 1 of this study reflect objectbased multisensory interactions arising from natural associations between visual and olfactory features of objects (e.g., orange odour and orange fruit image). Interestingly, odours enhanced discrimination of matching greyscale objects, indicating a connection between odours and visual object identity that is not dependent on colour. This is in agreement with our previous study that found odours had no effect on evoked responses to objects matching in colour but not form (Robinson et al., 2015). In contrast, various studies have found colours enhance olfactory discrimination (Dematte et al., 2009), indicating a major difference between the two types of olfactory-visual integration. It is possible that visualdriven olfactory enhancement is more reliant on semantic or conceptual representations, whereas olfactory-driven visual enhancement is object-based and involves specialised associations between odours and visual forms. To our knowledge, there is no evidence to suggest that males and females differ in their sensitivity to semantic or conceptual priming, indicating that our observed effects arose from direct multisensory processing rather than conceptual priming or imagery.

The results of the current study reveal that a matching odour can reliably improve detection of a masked image in females. This indicates an important role for olfaction in visual processing and suggests that odours influence a discrimination stage of object recognition. Importantly, our study shows that interactions between olfaction and vision manifest differently in males and females. Future research could use neuroimaging methods to determine where and when in the brain integration between olfactory and visual information occurs and how this differs between males and females.

Acknowledgments The study was funded by the Queensland Brain Institute and a UQ Major Equipment Grant to JR and JBM (RM2010002633). JBM was supported by an Australian Research Council (ARC) Australian Laureate Fellowship (FL110100103) and the ARC Centre of Excellence for Integrative Brain Function (ARC Centre Grant CE140100007). We thank Adam Bulley, Sarah Catchpoole, and Freya Young for their help with data collection and David Lloyd for his technical assistance. The authors declare no competing financial interests.

\section{References}

Andersson, L., Lundberg, C., Astrom, J., \& Nordin, S. (2011). Chemosensory attention, habituation and detection in women and men. International Journal of Psychophysiology, 79, 316-322. doi:10.1016/j.ijpsycho.2010.11.008

Bakeman, R. (2005). Recommended effect size statistics for repeated measures designs. Behavior Research Methods, 37(3), 379-384. doi:10.3758/BF03192707

Boehler, C. N., Schoenfeld, M. A., Heinze, H. J., \& Hopf, J. M. (2008). Rapid recurrent processing gates awareness in primary visual cortex.
Proceedings of the National Academy of Sciences of the United States of America, 105(25), 8742-8747. doi:10.1073/pnas.0801999105

Bushdid, C., Magnasco, M. O., Vosshall, L. B., \& Keller, A. (2014). Humans can discriminate more than 1 trillion olfactory stimuli. Science, 343(6177), 1370-1372. doi:10.1126/science.1249168

Carlson, T. A., Rauschenberger, R., \& Verstraten, F. A. J. (2007). No representation without awareness in the lateral occipital cortex. Psychological Science, 18(4), 298-302. doi:10.1111/j.14679280.2007.01892.x

Chen, K., Zhou, B., Chen, S., He, S., \& Zhou, W. (2013). Olfaction spontaneously highlights visual saliency map. Proceedings of the Royal Society B: Biological Sciences, 280(1768), 20131729. doi:10.1098/rspb.2013.1729

Choudhury, E. S., Moberg, P., \& Doty, R. L. (2003). Influences of age and sex on a microencapsulated odor memory test. Chemical Senses, 28(9), 799-805. doi:10.1093/chemse/bjg072

Cometto-Muniz, J. E., \& Abraham, M. H. (2008). Human olfactory detection of homologous n-alcohols measured via concentrationresponse functions. Pharmacology Biochemistry and Behavior, 89(3), 279-291. doi:10.1016/j.pbb.2007.12.023

Dematte, M. L., Sanabria, D., \& Spence, C. (2006). Cross-modal associations between odors and colors. Chemical Senses, 31(6), 531-538. doi:10.1093/chemse/bjj057

Dematte, M. L., Sanabria, D., \& Spence, C. (2009). Olfactory Discrimination: When Vision Matters? Chemical Senses, 34(2), 103-109. doi:10.1093/Chemse/Bjn055

Di Lollo, V., Enns, J. T., \& Rensink, R. A. (2000). Competition for consciousness among visual events: the psychophysics of reentrant visual processes. Journal of Experimental Psychology: General, 129(4), 481-507. doi:10.1037/0096-3445.129.4.481

Dienes, Z. (2011). Bayesian Versus Orthodox Statistics: Which Side Are You On? Perspectives on Psychological Science, 6(3), 274-290. doi:10.1177/1745691611406920

Dux, P. E., Visser, T. A., Goodhew, S. C., \& Lipp, O. V. (2010). Delayed reentrant processing impairs visual awareness: an objectsubstitution-masking study. Psychological Science, 21(9), 12421247. doi:10.1177/0956797610379866

Enns, J. T., \& Di Lollo, V. (1997). Object Substitution: A New Form of Masking in Unattended Visual Locations. Psychological Science, 8(2), 135-139. doi:10.1111/j.1467-9280.1997.tb00696.x

Fairweather, H. (1976). Sex differences in cognition. Cognition, 4(3), 231-280. doi:10.1016/0010-0277(76)90019-6

Filmer, H. L., Mattingley, J. B., \& Dux, P. E. (2015). Object Substitution Masking for an Attended and Foveated Target. Journal of Experimental Psychology: Human Perception and Performance, 41(1), 6-10. doi:10.1037/xhp0000024

Frasnelli, J., Lundstrom, J. N., Boyle, J. A., Djordjevic, J., Zatorre, R. J., \& Jones-Gotman, M. (2010). Neuroanatomical correlates of olfactory performance. Experimental Brain Research, 201(1), 1-11. doi:10.1007/s00221-009-1999-7

Garcia-Falgueras, A., Junque, C., Gimenez, M., Caldu, X., Segovia, S., \& Guillamon, A. (2006). Sex differences in the human olfactory system. Brain Research, 1116(1), 103-111. doi:10.1016/j. brainres.2006.07.115

Gilbert, A. N., Martin, R., \& Kemp, S. E. (1996). Cross-modal correspondence between vision and olfaction: the color of smells. American Journal of Psychology, 109(3), 335-351. doi:10.2307/1423010

Goodhew, S. C., Pratt, J., \& Dux, P. E. (2013). Substituting objects from consciousness : A review of object substitution masking. Psychonomic Bulletin and Review, 20, 859-877. doi:10.3758/s13423-013-0400-9

Goodhew, S. C., Visser, T. A., Lipp, O. V., \& Dux, P. E. (2011). Implicit semantic perception in object substitution masking. Cognition, 118(1), 130-134. doi:10.1016/j.cognition.2010.10.013

Gottfried, J. A., \& Dolan, R. J. (2003). The Nose Smells What the Eye Sees: Crossmodal Visual Facilitation of Human Olfactory 
Perception. Neuron, 39(2), 375-386. doi:10.1016/S0896-6273(03 00392-1

Grefkes, C., \& Fink, G. R. (2005). The functional organization of the intraparietal sulcus in humans and monkeys. Journal of Anatomy, 207(1), 3-17. doi:10.1111/j.1469-7580.2005.00426.x

Habel, U., Koch, K., Pauly, K., Kellermann, T., Reske, M., Backes, V., ... Schneider, F. (2007). The influence of olfactory-induced negative emotion on verbal working memory: Individual differences in neurobehavioral findings. Brain Research, 1152, 158-170. 10.1016/J. Brainres.2007.03.048

Hanson-Vaux, G., Crisinel, A. S., \& Spence, C. (2013). Smelling shapes: crossmodal correspondences between odors and shapes. Chemical Senses, 38(2), 161-166. doi:10.1093/chemse/bjs087

Harris, J. A., Ku, S., \& Woldorff, M. G. (2013). Neural processing stages during object-substitution masking and their relationship to perceptual awareness. Neuropsychologia, 51(10), 1907-1917. doi:10.1016 /j.neuropsychologia.2013.05.023

Haxby, J. V., Hoffman, E. A., \& Gobbini, M. I. (2000). The distributed human neural system for face perception. Trends in Cognitive Sciences, 4(6), 223-233. doi:10.1016/s1364-6613(00)01482-0

Heath, M., Maraj, A., Godbolt, B., \& Binsted, G. (2008). Action Without Awareness : Reaching to an Object You Do Not Remember Seeing. PLoS ONE, 3(10), e3539. doi:10.1371/journal.pone.0003539

Ho, C., \& Spence, C. (2005). Olfactory facilitation of dual-task performance. Neuroscience Letters, 389(1), 35-40. doi:10.1016/j. neulet.2005.07.003

Jeffreys, H. (1961). Theory of probability (Third). Oxford: Oxford University Press. Retrieved from http://books.google.ca/books?id= vh9Act9rtzQC\&printsec $=$ frontcover $\# \mathrm{v}=$ onepage \&q \& $\mathrm{f}=$ false

Kemp, S. E., \& Gilbert, A. N. (1997). Odor intensity and color lightness are correlated sensory dimensions. American Journal of Psychology, 110(1), 35-46. doi:10.2307/1423699

Koelega, H. S. (1970). Extraversion, sex, arousal and olfactory sensitivity. Acta Psychologica, 34(1), 51-66. doi:10.1016/0001-6918(70)90004-1

Lakens, D. (2013). Calculating and reporting effect sizes to facilitate cumulative science: a practical primer for -tests and ANOVAs. Frontiers in Psychology, 4, 863. doi:10.3389/fpsyg.2013.00863

Larsson, M., Nilsson, L. G., Olofsson, J. K., \& Nordin, S. (2004). Demographic and cognitive predictors of cued odor identification: evidence from a population-based study. Chemical Senses, 29(6), 547-554. doi:10.1093/chemse/bjh059

Lleras, A., \& Moore, C. M. (2003). When the Target Becomes the Mask : Using Apparent Motion to Isolate the Object-Level Component of Object Substitution Masking. Journal of Experimental Psychology. Human Perception and Performance, 29(1), 106-120. doi:10.1037 /0096-1523.29.1.106

Lundstrom, J. N., \& Hummel, T. (2006). Sex-specific hemispheric differences in cortical activation to a bimodal odor. Behavioural Brain Research, 166(2), 197-203. doi:10.1016/j.bbr.2005.07.015

Morey, R. D., \& Rouder, J. N. (2011). Bayes factor approaches for testing interval null hypotheses. Psychological Methods, 16(4), 406-419. doi:10.1037/a0024377

Oberg, C., Larsson, M., \& Backman, L. (2002). Differential sex effects in olfactory functioning: the role of verbal processing. Journal of the International Neuropsychological Society, 8(5), 691-698. doi:10.1017/s1355617702801424

Osterbauer, R. A., Matthews, P. M., Jenkinson, M., Beckmann, C. F., Hansen, P. C., \& Calvert, G. A. (2005). Color of scents: chromatic stimuli modulate odor responses in the human brain. Journal of Neurophysiology, 93(6), 3434-3441. doi:10.1152/jn.00555.2004

Pilling, M., \& Gellatly, A. (2010). Object substitution masking and the object updating hypothesis. Psychonomic Bulletin and Review, 17(5), 737-742. doi:10.3758/PBR.17.5.737

Reiss, J. E., \& Hoffman, J. E. (2007). Disruption of early face recognition processes by object substitution masking. Visual Cognition, 15(7), 789-798. doi:10.1080/13506280701307035
Robinson, A. K., Mattingley, J. B., \& Reinhard, J. (2013). Odors enhance the salience of matching images during the attentional blink. Frontiers in Integrative Neuroscience, 7, 77. doi:10.3389 /fnint.2013.00077

Robinson, A. K., Reinhard, J., \& Mattingley, J. B. (2015). Olfaction Modulates Early Neural Responses to Matching Visual Objects. Journal of Cognitive Neuroscience, 27(4), 832-841. doi:10.1162 /jocn a 00732

Sabatinelli, D., Flaisch, T., Bradley, M. M., Fitzsimmons, J. R., \& Lang, P. J. (2004). Affective picture perception: gender differences in visual cortex? Neuroreport, 15(7), 1109-1112. doi:10.1097 /00001756-200405190-00005

Sakamoto, R., Minoura, K., Usui, A., Ishizuka, Y., \& Kanba, S. (2005). Effectiveness of Aroma on Work Efficiency: Lavender Aroma during Recesses Prevents Deterioration of Work Performance. Chemical Senses, 30(8), 683-691. doi:10.1093/chemse/bji061

Seo, H. S., Arshamian, A., Schemmer, K., Scheer, I., Sander, T., Ritter, G., \& Hummel, T. (2010). Cross-modal integration between odors and abstract symbols. Neuroscience Letters, 478(3), 175-178. doi:10.1016/j.neulet.2010.05.011

Seo, H. S., Roidl, E., Muller, F., \& Negoias, S. (2010). Odors enhance visual attention to congruent objects. Appetite, 54(3), 544-549. doi:10.1016/j.appet.2010.02.011

Sergent, J., Ohta, S., \& Macdonald, B. (1992). Functional Neuroanatomy of Face and Object Processing. Brain, 115(1), 15-36. doi:10.1093 /brain/115.1.15

Seubert, J., Freiherr, J., Frasnelli, J., Hummel, T., \& Lundstrom, J. N. (2013). Orbitofrontal cortex and olfactory bulb volume predict distinct aspects of olfactory performance in healthy subjects. Cerebral Cortex, 23(10), 2448-2456. doi:10.1093/cercor/bhs230

Stanislaw, H., \& Todorov, N. (1999). Calculation of signal detection theory measures. Behavior Research Methods, Instruments and Computers, 31(1), 137-149. doi:10.3758/BF03207704

Warm, J. S., Dember, W. N., \& Parasuraman, R. (1991). Effects of Olfactory Stimulation on Performance and Stress in a Visual Sustained Attention Task. Journal of the Society of Cosmetic Chemists, 42(3), 199-210. Retrieved from http://journal.scconline. org//pdf/cc1991/cc042n03/p00199-p00210.pdf

Wedekind, C., \& Furi, S. (1997). Body odour preferences in men and women: do they aim for specific MHC combinations or simply heterozygosity? Proceedings of the Royal Society B: Biological Sciences, 264, 1471-1479. doi:10.1098/rspb.1997.0204

Wedekind, C., Seebeck, T., Bettens, F., \& Paepke, A. (1995). MHCdependent mate preferences in humans. Proceedings of the Royal Society B: Biological Sciences, 260(1359), 245-249. doi:10.1098 /rspb.1995.0087

Zampini, M., \& Spence, C. (2012). Assessing the Role of Visual and Auditory Cues in Multisensory Perception of Flavor. In M. M. Murray \& M. T. Wallace (Eds.), The Neural Bases of Multisensory Processes. Boca Raton, FL: CRC Press. Retrieved from http://www. ncbi.nlm.nih.gov/pubmed/22593877

Zellner, D. A., \& Kautz, M. A. (1990). Color affects perceived odor intensity. Journal of Experimental Psychology: Human Perception and Performance, 16(2), 391-397. doi:10.1037/00961523.16.2.391

Zhou, W., Jiang, Y., He, S., \& Chen, D. (2010). Olfaction Modulates Visual Perception in Binocular Rivalry. Current Biology, 20(15), 1356-1358. doi:10.1016/j.cub.2010.05.059

Zhou, W., Zhang, X., Chen, J., Wang, L., \& Chen, D. (2012). Nostrilspecific olfactory modulation of visual perception in binocular rivalry. Journal of Neuroscience, 32(48), 17225-17229. doi:10.1523 jneurosci.2649-12.2012

Zucco, G. M., Aiello, L., Turuani, L., \& Koster, E. (2012). Odor-evoked autobiographical memories: age and gender differences along the life span. Chemical Senses, 37(2), 179-189. doi:10.1093 /chemse/bjr089 